\title{
Comprehensive managements of metastatic renal tumor with Mayo III inferior vena cava tumor thrombus: a case report
}

\author{
Chenkui Miao $^{1 \#}$, Yuhao Wang ${ }^{1 \#}$, Chao Hou ${ }^{1 \#}$, Wen Chen ${ }^{2}$, Aiming Xu ${ }^{1}$, Zengjun Wang ${ }^{1}$ \\ ${ }^{1}$ Department of Urology, ${ }^{2}$ Department of Pathology, The First Affiliated Hospital of Nanjing Medical University, Nanjing 210029, China \\ \#These authors contributed equally to this work. \\ Correspondence to: Zengjun Wang. Department of Urology, The First Affiliated Hospital of Nanjing Medical University, Nanjing 210029, China. \\ Email: zengjunwang@njmu.edu.cn.
}

\begin{abstract}
Renal tumor with inferior vena cava (IVC) tumor thrombus still remains one of the most medical challenges in urological oncology. Despite numerous researches reporting the surgical experiences and survivals of this kind of patients, there is still lacking a standard recommended therapy right now. We reported a case of metastatic renal cell carcinoma with Mayo III IVC tumor thrombus who underwent robotic-assisted complete removal of the intracaval thrombus and radical left nephrectomy followed by renal arterial chemoembolization and pazopanib administration. It provides a new scheme and mode of diagnosis and treatment for this kind of patients. The patient was a 50-year-old man with left low-back pain for 20 days diagnosed with left renal tumor and Mayo III IVC tumor thrombus at the earliest. Initially, the patient underwent the renal arterial chemoembolization and targeted treatment to inhibit tumor's progression. After a two-year therapy period, the size of renal mass and lung nodules decreased than before, as well as the IVC tumor thrombus dropped to level II. Considering the efficacy of previous treatments, we performed robot-assisted IVC thrombectomy and radical left nephrectomy for this patient. The postoperative pathological examination confirmed the diagnosis of tumor thrombus as renal clear cell carcinoma. The patients recovered well after surgery and was followed-up for 36 months during the whole treatment course. This case with metastatic renal cell carcinoma (mRCC) and Mayo III IVC tumor thrombus received the interventional therapy, molecular targeted therapy and robot-assisted surgery successively, and acquired satisfying outcome. Patients with mRCC always suffer shorter overall survivals and aggressive progression compared with those localized tumors, therefore it is essential to formulate rational comprehensive treatment and carry out in time following-up.
\end{abstract}

Keywords: Renal carcinoma; venous tumor thrombus; molecular targeted therapy; robotic surgery; case report

Submitted Oct 04, 2019. Accepted for publication Dec 12, 2019.

doi: $10.21037 /$ tau.2019.12.13

View this article at: http://dx.doi.org/10.21037/tau.2019.12.13

\section{Introduction}

Renal cell carcinoma (RCC) is one of the most common malignant tumors in the urinary system, accounting for about $2-3 \%$ of adult malignancies. RCC not only invades adjacent fatty organs, but also tends to spread into veins, forming tumor thrombus. $4 \%$ to $10 \%$ of the local advanced renal carcinoma are fused with venous propagation into the renal vein and the inferior vena cava (IVC) tumor thrombus, among which the left renal tumor is relatively rare (1). IVC tumor thrombus is classified into four levels named as 0 , I, II, III and IV, depending on the position of thrombus in relation to the hepatic veins (2). Previous surgical management of patients with IVC tumor thrombus originating from renal tumor usually included complete IVC thrombectomy, radical nephrectomy (RN), and ipsilateral retroperitoneal lymphadenectomy (RPLND) under the open operative condition. During this complex process, it requires a large muscle-cutting abdominal or thoracoabdominal incision, the control of IVC 

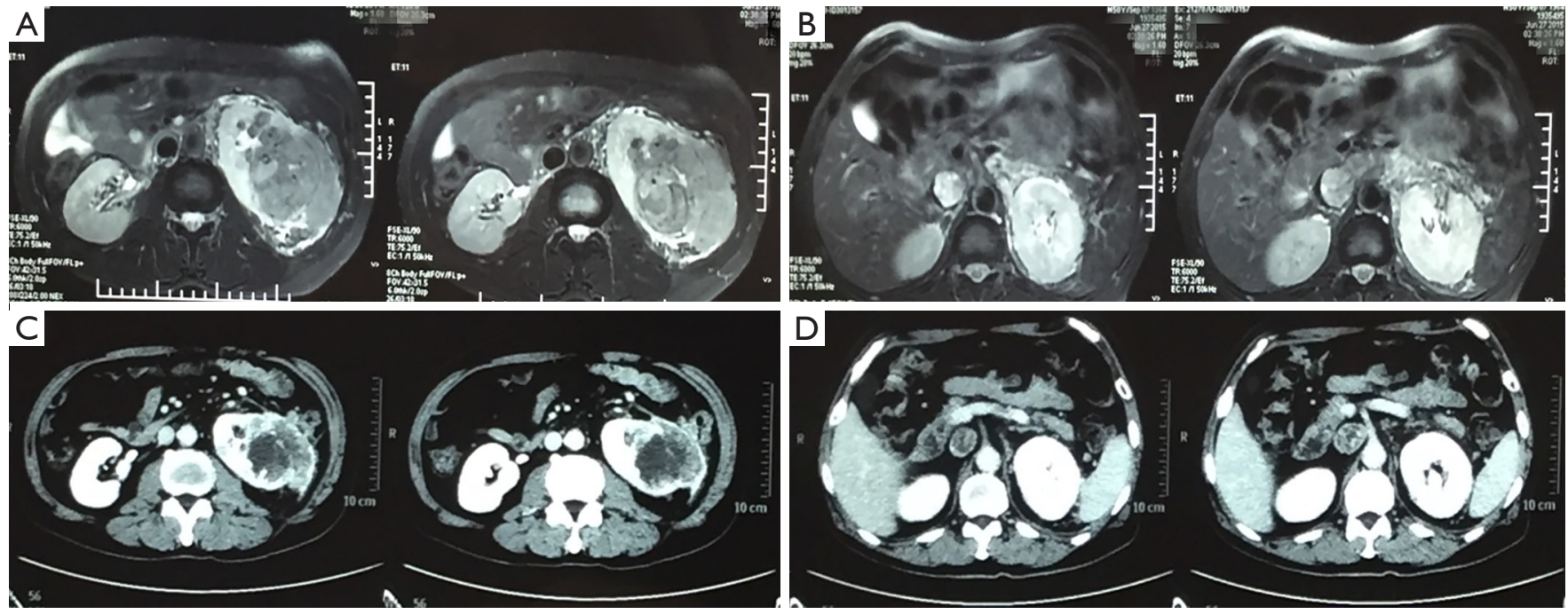

Figure 1 MRI examination showed left kidney and IVC mass before and after TKI therapy. (A) Left kidney mass before TKI therapy; (B) IVC mass before TKI therapy; (C) left kidney mass after TKI therapy; (D) IVC mass after TKI therapy. IVC, inferior vena cava; TKI, tyrosine kinase inhibitor.

neoplastic emboli as well as the prevention of thrombus fragmentation (3). Advancement has arrived when the minimally invasive IVC tumor thrombectomy appeared recently, for example the laparoscopic or robotic assisted surgery (4-6).

However, for patients with higher IVC thrombus level (III, IV), surgical management is still challenging because of high death risk. A growing evidence has illuminated the safety and efficacy of presurgical molecular-targeting agents for non-metastatic or metastatic tumor (7-9) and tumor thrombus reaches into the IVC $(10,11)$. Advances in neoadjuvant settings were shown to significantly extend the survival in metastatic RCC over the past decade. These kinds of molecular-targeting drugs include sunitinib, pazopanib and sorafenib, among which were proposed as potential candidates for preoperative application of IVC tumor thrombus $(11,12)$. Herein, we present a 50-year-old mRCC patient with Mayo III IVC tumor thrombus, which means the surgery has been lost. The patient underwent robotic-assisted complete removal of the intracaval thrombus and radical left nephrectomy followed by renal arterial chemoembolization and pazopanib administration. Through the series of comprehensive treatment, the patient is now in a stable state. We reported the case so that this comprehensive therapy can provide a new scheme and mode of diagnosis and treatment for this kind of patients.

\section{Case presentation}

A 50-year-old man with left low-back pain for 20 days went to the local hospital and received a preliminary examination. B-ultrasound and magnetic resonance imaging (MRI) examination showed an approximately $10 \mathrm{~cm} \times 8.3 \mathrm{~cm}$ mass in the left kidney, combined with a $3.6 \mathrm{~cm} \times 2.6 \mathrm{~cm}$ mass extending to left renal vein and IVC (Figure 1A,B). Chest computed tomography (CT) examination indicated multiple nodules in both lungs, more likely to be metastasis. According to the thrombus level system mentioned above, IVC thrombus of this patient was at level III. Considering the patient developed a metastatic renal cancer and Mayo III IVC tumor thrombus, it was of high risk and low effectivity to perform surgical treatment at present. The patient denied a previous history of cancer and a family history of similar tumors and expressed that he went to our hospital as soon as getting the examination. No positive results were found in the physical examination.

According to the history and the examination, it is a definitive diagnosis that the patient developed a metastatic renal cancer and Mayo III IVC tumor thrombus. The patient was willing to accept the comprehensive treatment after being told the potential injury and risk of such a treatment.

Therefore, the patient underwent the left renal artery chemoembolization in our hospital and took pazopanib orally regularly $800 \mathrm{mg}$ once a day without food for nearly two years. During the therapy course, there was no obvious 

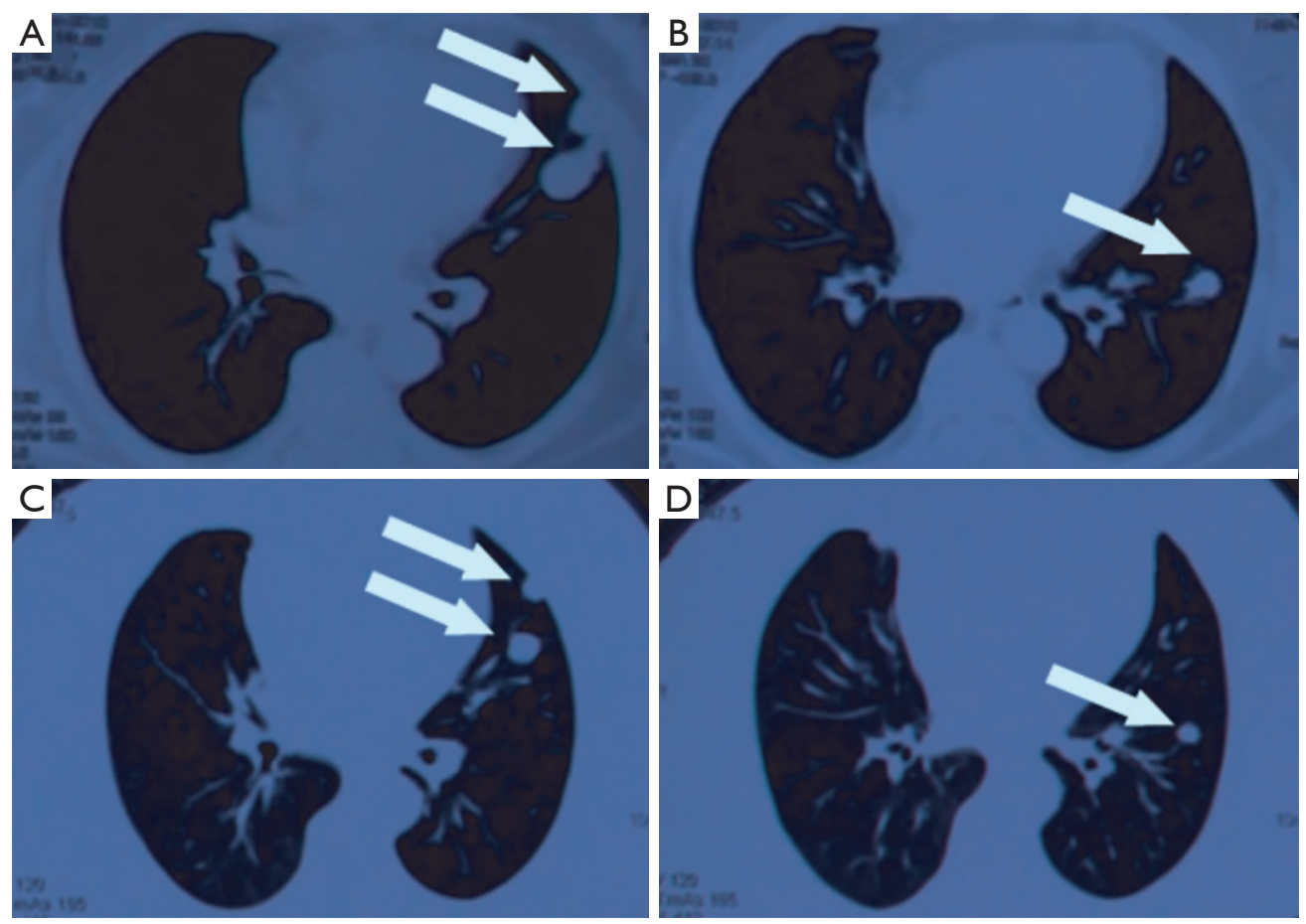

Figure 2 CT examination indicated multiple nodules in both lungs before and after TKI therapy. (A,B) Lung nodules before TKI therapy; (C,D) lung nodules after TKI therapy. The arrows mean lung nodules. TKI, tyrosine kinase inhibitor.

adverse reactions. CT examination two years later showed that the tumor size decreased to about $9.2 \mathrm{~cm} \times 6.5 \mathrm{~cm}$, and the tumor thrombus in the inferior vena cava turned to level II (Figure 1C,D). Simultaneously, the metastatic lesions in the lung also obviously reduced as compared to before (Figure 2).

Considering the patient was in a stable condition, tumor thrombus levels fell down from III to II, and renal tumor with lung metastases became narrow than prior therapy, we performed robot-assisted IVC thrombectomy and radical left nephrectomy for the patient after 2 weeks Pazopanib discontinuation and left renal artery embolization one day before operation. The whole surgery procedure was as follows: (I) free inferior vena cava branch, including genital vein, hepatic accessory vein, lumbar vein and adrenal vein, which should be carefully blocked in turn using Hemolock after separation (Figure $3 A, B$ ); (II) free inferior vena cava distal and proximal, left and right renal veins (Figure 3C,D); (III) sequentially block distal vena cava, right renal vein, and proximal vena cava (Figure 3E,F,G); (IV) use the EndoGIA to disconnect the left renal vein (Figure $3 H$ ); (V) dissect the inferior vena cava, remove the tumor thrombus completely (Figure 3I,7); (VI) Suture the inferior vena cava wall continuously (Figure $3 K, L$ ), then open the bloodstream and perform the radical nephrectomy. The operation went well, the left kidney and tumor thrombus were removed completely (Figure 4A,B).

Postoperative pathology examination confirmed the diagnosis of dissected renal tumor to be clear cell carcinoma, Furhman II to III with necrosis, inferior vena cava tissue to be tumor thrombus with necrosis (Figure $4 C, D$ ). The patient continued to receive targeted therapy after hospital discharge and were followed up regularly. Currently, the lesion of lung was stable and patient was generally in good condition during the 36-month follow-up period.

\section{Discussion}

RCC can not only invade adjacent adipose organs, but also easily spread into veins to form tumor thrombi. Due to the short right renal vein, it is more likely to fall off to the inferior vena cava after forming tumor thrombus. Therefore, most renal cell carcinoma with tumor thrombus is found in the right kidney cancer, while the left kidney cancer with tumor thrombus is relatively rare. After the formation of vena cava carcinoma thrombi in the left renal vein, it is easier to establish multi-branch collateral 

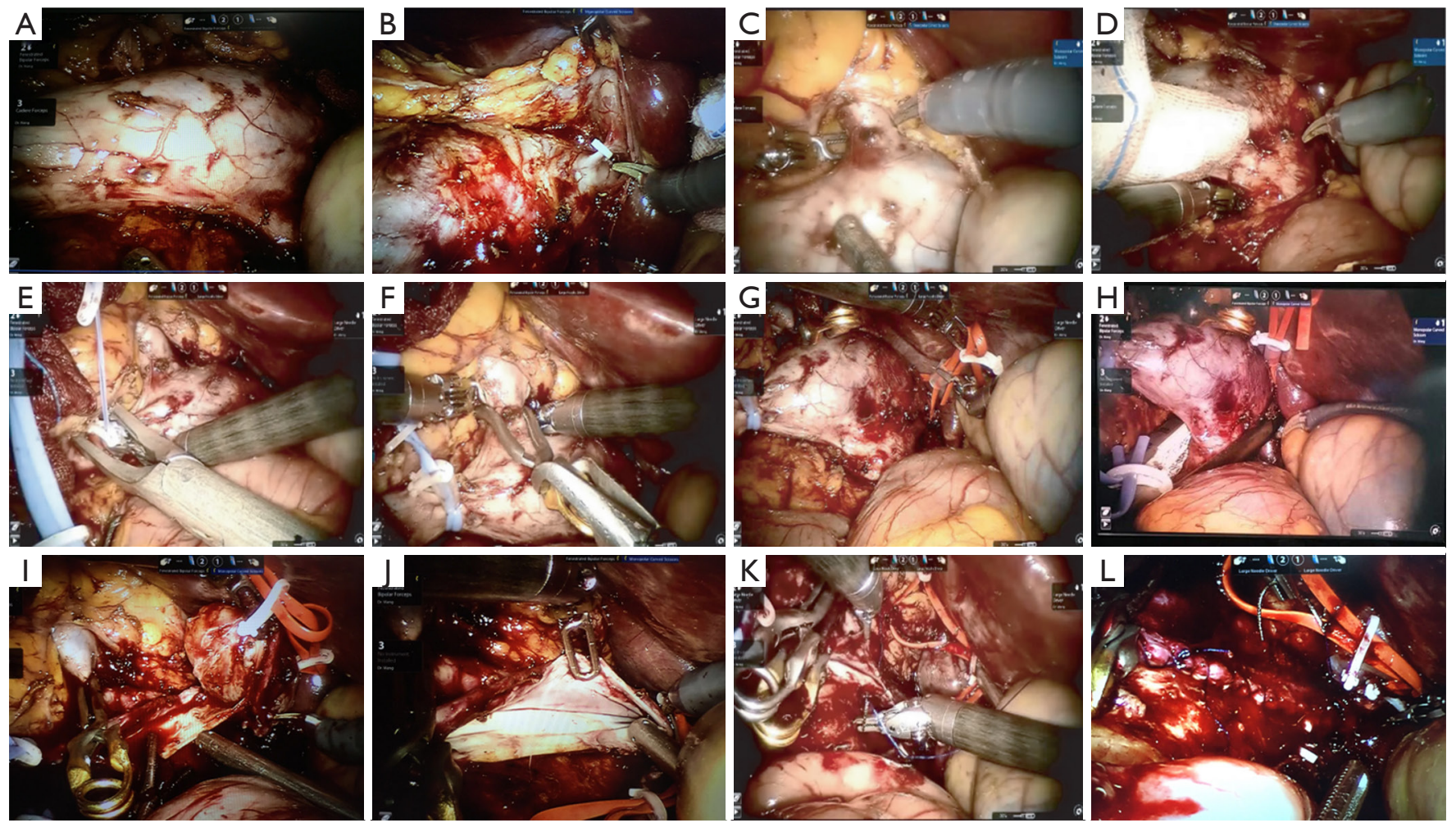

Figure 3 Process of robotic-assisted IVC thrombectomy. (A,B) Free inferior vena cava branch, including genital vein, hepatic accessory vein, lumbar vein and adrenal vein; (C,D) free inferior vena cava distal and proximal, left and right renal veins; (E,F,G) sequentially block distal vena cava, right renal vein, and proximal vena cava $(\mathrm{E}, \mathrm{F}, \mathrm{G}) ;(\mathrm{H})$ use Endo-GIA to disconnect the left renal vein; $(\mathrm{I}, \mathrm{J})$ dissect the inferior vena cava, remove the tumor thrombus completely; $(\mathrm{K}, \mathrm{L})$ suture the inferior vena cava wall continuously. IVC, inferior vena cava.

circulation. The formation of long-term collateral circulation makes the renal perivascular hypertrophy and distention, and the intraoperative wound is prone to extensive bleeding. In addition, during the operation, patients need to change from the left lateral position to the right lateral position, which makes the operation more difficult (13).

Radical nephrectomy and tumor thrombectomy of inferior vena cava is a traditional and effective treatment for renal cancer with inferior vena cava tumor thrombus, but the open surgery has greater trauma and slow postoperative recovery (14). Although laparoscopic technology is becoming more and more mature, laparoscopic radical nephrectomy with the inferior vena cava tumor thrombectomy is still one of the most challenging operations in the field of urology. Recently, cases with lower grade of Mayo vein tumor thrombectomy were mostly selected for complete laparoscopic tumor thrombectomy of inferior vena cava (15). At present, it is relatively rare to report the treatment of left renal cancer with Mayo III IVCTT.

At present, a variety of targeted drugs have been approved for the treatment of renal cancer, which can be roughly divided into: tyrosine kinase inhibitors (TKIs), vascular epidermal growth factor (VEGF) monoclonal antibodies, and mammalian target of rapamycin (mTOR) inhibitors according to the different targets of targeted drugs. TKI drugs have multiple effects such as inhibiting tumor angiogenesis and anti-tumor cell growth. The representative drugs include sunitinib, sorafenib, pazopanib and axitinib.

For patients with IVCTT, whether targeted drugs should be applied preoperatively or not varies greatly among the literature reports. Some scholars believe that the preoperative use of targeted drugs can improve the prognosis of patients, reduce the level of carcinoma thrombus and reduce the difficulty of surgery. Shuch et al. reported the successful renal cancer cases with high-level tumor thrombus, whom were treated with molecular targeted therapy before surgery, and then radical 

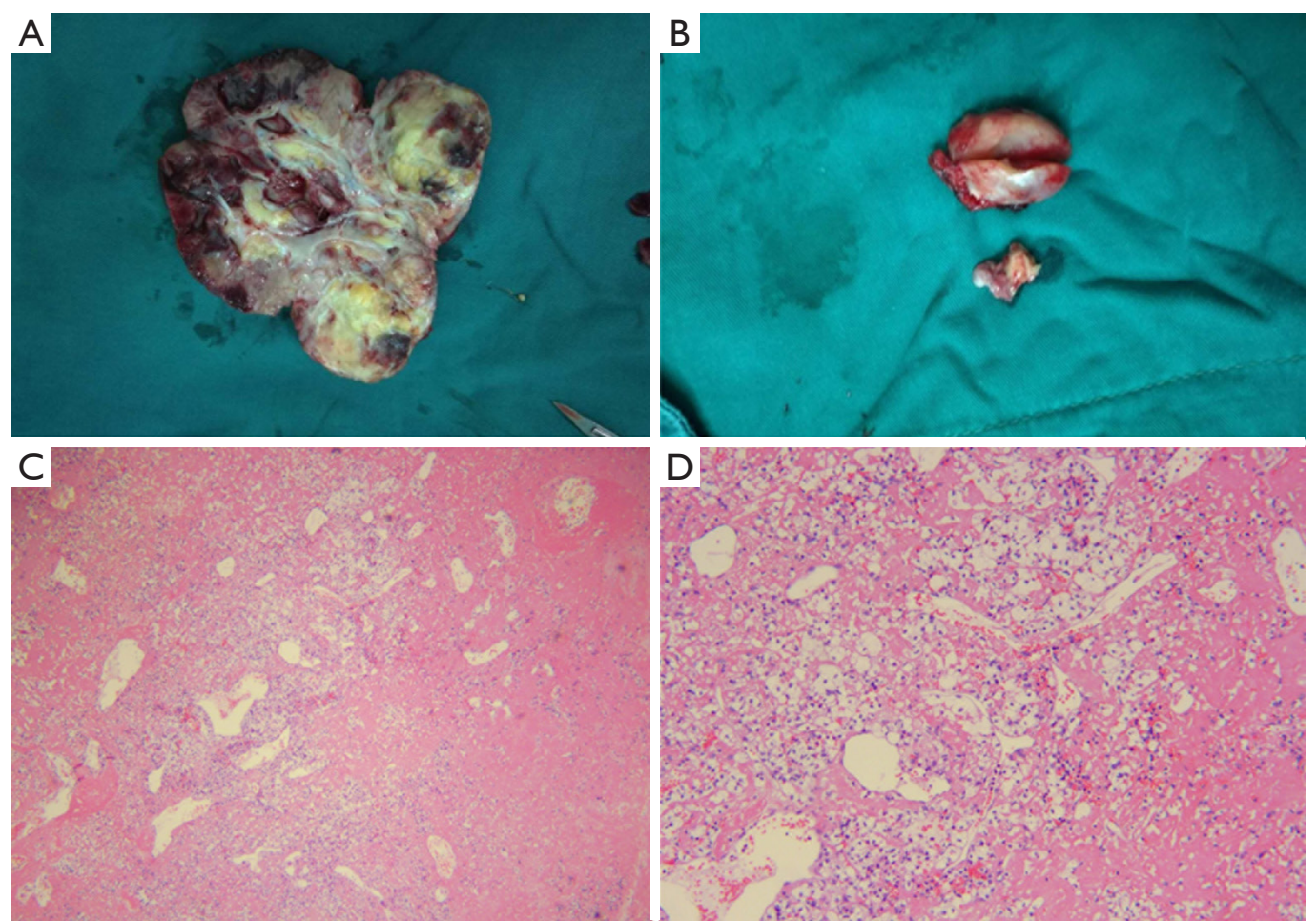

Figure 4 Dissected left kidney, IVC tumor and H\&E staining. (A) Left kidney; (B) IVC tumor; (C,D) H\&E staining of IVC tumor. C, 40×; D, 100x. IVC, inferior vena cava.

nephrectomy and inferior vena cava tumor thrombectomy were performed after tumor thrombus was degraded (16). Statistics show that the degradation of tumor thrombus accounted for $5-44 \%$, the steady change accounted for 28 $91 \%$, and the progression accounted for $5-28 \%$ according to the past reports on using targeted drugs. Psutka $e t$ al. thought that early surgery was not recommended for patients with major surgical risks or distant metastasis, and first-line targeted drug therapy could be given firstly (17). Pazopanib, approved in October 2009 by U.S. Food and Drug Administration (FDA), is a new kind of tyrosine kinase inhibitors functioning by inhibiting the activity of VEGFR, PDGFR and c-KIT. Studies have shown that pazopanib and sunitinib have similar anticancer effects on the patients with advanced renal cancer, with relatively small adverse effects and high quality of life (18). After a full evaluation of the patient's condition and considering the request of the patient and his family members for active treatment, we treated the patient with pazopanib, and then controlled the local lesion by interventional embolization chemotherapy. Following up the patient for 2 years, we found that the left renal tumor decreased significantly, as well as the level of IVCTT degraded from III to II. Therefore, we decided to perform robotic assisted laparoscopic radical nephrectomy combined with thrombectomy of inferior vena cava tumor. Due to the heavy adhesion around the tumor after taking targeted drugs, which increases the difficulty of surgery, it is necessary to discontinue the drugs for more than 2 weeks before surgery $(11,19)$.

Because it is difficult to expose the renal artery through abdominal surgery, and the existence of tumor thrombus makes it more difficult. Preoperative renal artery embolization can reduce bleeding and shorten the operation time during nephrectomy. Meanwhile, it can prevent intraoperative perfusion pressure from causing the movement of the tumor thrombus. Therefore, the renal artery was embolized the day before radical nephrectomy in this case. During the operation, it was found that the pulsation of the left renal artery was significantly weakened, that the perirenal fat formed edema zone, and that the kidney and tumor texture were relatively soft, which was easy to completely remove. The degree of tumor thrombus entering the vena cava does not change the prognosis of patients $(20,21)$, and the invasion of the inferior vena cava wall is a risk factor for disease recurrence and poor prognosis (22). No invasion of the inferior vena cava wall 
was found in this case, so the inferior vena cava wall was not resected.

In this case, we reported the comprehensive management of a renal tumor patient with IVC tumor thrombus and multiple metastases. The patient received robotic-assisted $\mathrm{RN}$ and IVC tumor thrombectomy after preoperative neoadjuvant targeted therapy, and obtained considerable disease remission during the follow-up. Therefore, neoadjuvant therapy based on molecular targeted drugs plays a key role in the comprehensive management of advanced RCC patients with IVC tumor thrombus. It can reduce the tumor size, operation difficulty, and enable patients with advanced renal cancer to obtain surgery probability. However, the case we have reported has some limitations. Firstly, we reported too few cases, just one case, because it is very difficult to meet such patients. Secondly, the effectiveness of this combinative treatment remains to be seen or to be summarized by expanding the cases and the follow-up time.

\section{Acknowledgments}

Funding: The study was supported by the National Natural Science Foundation of China (Grants nos. 81270685 and 81771640); Project of Nanjing Science and Technology Committee (201605001); Postgraduate Research \& Practice Innovation Program of Jiangsu Province (KYCX19_1159); International Exchange and Cooperation Program for Postgraduates of Nanjing Medical University; and Postgraduate Research \& Practice Innovation Program of Jiangsu Province (KYCX19_1159)

\section{Footnote}

Conflicts of Interest: All authors have completed the ICMJE uniform disclosure form (available at http://dx.doi. org/10.21037/tau.2019.12.13). The authors have no conflicts of interest to declare.

Ethical Statement: The authors are accountable for all aspects of the work in ensuring that questions related to the accuracy or integrity of any part of the work are appropriately investigated and resolved. Written informed consent was obtained from the patient for publication of this manuscript and any accompanying images.

Open Access Statement: This is an Open Access article distributed in accordance with the Creative Commons
Attribution-NonCommercial-NoDerivs 4.0 International License (CC BY-NC-ND 4.0), which permits the noncommercial replication and distribution of the article with the strict proviso that no changes or edits are made and the original work is properly cited (including links to both the formal publication through the relevant DOI and the license). See: https://creativecommons.org/licenses/by-nc$\mathrm{nd} / 4.0 /$.

\section{References}

1. Blute ML, Leibovich BC, Lohse CM, et al. The Mayo Clinic experience with surgical management, complications and outcome for patients with renal cell carcinoma and venous tumour thrombus. BJU Int 2004;94:33-41.

2. Neves RJ, Zincke H. Surgical treatment of renal cancer with vena cava extension. Br J Urol 1987;59:390-5.

3. Chopra S, Simone G, Metcalfe C, et al. Robot-assisted Level II-III Inferior Vena Cava Tumor Thrombectomy: Step-by-Step Technique and 1-Year Outcomes. Eur Urol 2017;72:267-74.

4. Gill IS, Metcalfe C, Abreu A, et al. Robotic level III inferior vena cava tumor thrombectomy: initial series. J Urol 2015;194:929-38.

5. Bratslavsky G, Cheng JS. Robotic-assisted radical nephrectomy with retrohepatic vena caval tumor thrombectomy (level III) combined with extended retroperitoneal lymph node dissection. Urology 2015;86:1235-40.

6. Shao P, Li J, Qin C, et al. Laparoscopic radical nephrectomy and inferior vena cava thrombectomy in the treatment of renal cell carcinoma. Eur Urol 2015;68:115-22.

7. Karam JA, Devine CE, Urbauer DL, et al. Phase 2 trial of neoadjuvant axitinib in patients with locally advanced nonmetastatic clear cell renal cell carcinoma. Eur Urol 2014;66:874-80.

8. Thomas AA, Rini BI, Lane BR, et al. Response of the primary tumor to neoadjuvant sunitinib in patients with advanced renal cell carcinoma. J Urol 2009;181:518-23.

9. Rini BI, Garcia J, Elson P, et al. The effect of sunitinib on primary renal cell carcinoma and facilitation of subsequent surgery. J Urol 2012;187:1548-54.

10. Kondo T, Hashimoto Y, Kobayashi H, et al. Presurgical targeted therapy with tyrosine kinase inhibitors for advanced renal cell carcinoma: clinical results and histopathological therapeutic effects. Jpn J Clin Oncol 2010;40:1173-9.

11. Cost NG, Delacroix SE Jr, Sleeper JP, et al. The impact 
of targeted molecular therapies on the level of renal cell carcinoma vena caval tumor thrombus. Eur Urol 2011;59:912-8.

12. Kwon T, Lee JL, You D, et al. Impact of surgery on the prognosis of metastatic renal cell carcinoma with IVC thrombus received TKI therapy. J Surg Oncol 2014;110:145-50.

13. Katkoori D, Murugesan M, Ciancio G, et al. Tumor thrombus involving the inferior vena cava in renal malignancy: is there a difference in clinical presentation and outcome among right and left side tumors? Int Braz J Urol 2009;35:652-6; discussion 656-7.

14. Kirkali Z, Van Poppel H. A critical analysis of surgery for kidney cancer with vena cava invasion. Eur Urol 2007;52:658-62.

15. Xu B, Zhao Q, Jin J, et al. Laparoscopic versus open surgery for renal masses with infrahepatic tumor thrombus: the largest series of retroperitoneal experience from China. J Endourol 2014;28:201-7.

16. Shuch B, Riggs SB, LaRochelle JC, et al. Neoadjuvant targeted therapy and advanced kidney cancer: observations and implications for a new treatment paradigm. BJU Int

Cite this article as: Miao C, Wang Y, Hou C, Chen W, Xu A, Wang Z. Comprehensive managements of metastatic renal tumor with Mayo III inferior vena cava tumor thrombus: a case report. Transl Androl Urol 2020;9(2):812-818. doi: 10.21037/ tau.2019.12.13
2008;102:692-6.

17. Psutka SP, Leibovich BC. Management of inferior vena cava tumor thrombus in locally advanced renal cell carcinoma. Ther Adv Urol 2015;7:216-29.

18. Motzer RJ, McCann L, Deen K. Pazopanib versus sunitinib in renal cancer. New Engl J Med 2013;369:1970.

19. Kroeger N, Gajda M, Zanow J, et al. Downsizing a tumor thrombus of advanced renal cell carcinoma with neoadjuvant systemic therapy and resulting histopathological effects. Urol Int 2010;84:479-84.

20. Haferkamp A, Bastian PJ, Jakobi H, et al. Renal cell carcinoma with tumor thrombus extension into the vena cava: prospective long-term followup. J Urol 2007;177:1703-8.

21. Pouliot F, Shuch B, LaRochelle JC, et al. Contemporary management of renal tumors with venous tumor thrombus. J Urol 2010;184:833-41; quiz 1235.

22. Hirono M, Kobayashi M, Tsushima T, et al. Impacts of clinicopathologic and operative factors on shortterm and long-term survival in renal cell carcinoma with venous tumor thrombus extension: a multi-institutional retrospective study in Japan. BMC Cancer 2013;13:447. 